\title{
Ubiquitous Intelligence and Computing: building smart environment in real and cyber space
}

\author{
Ching-Hsien Hsu
}

Published online: 29 October 2011

(C) Springer-Verlag 2011

Ubiquitous Intelligence and Computing (UIC) is emerging rapidly as an exciting new paradigm including technologies of pervasive computing, wireless communication and ambient intelligence to provide computing and communication services any time and anywhere. It usually refers to the creation and deployment of computing technology in such a way that it becomes an invisible part of the fabric of everyday life and commerce. As ubiquitous computing presents a new trend of information and communication technologies for connecting cyber and physical domains, in such era, computers in the traditional sense gradually fade from view. Namely, information and communication mediated by computers is available anywhere and anytime through devices that are embedded in our environment, completely inter-connected, intuitive, effortlessly portable and constantly available.

Ubiquitous sensors, devices, networks and information are paving the way towards a smart world in which computational intelligence is distributed throughout the physical environment to provide reliable and relevant services to people. This ubiquitous intelligence will change the computing landscape because it will enable new breeds of applications and systems to be developed and the realm of computing possibilities will be significantly extended. This special issue is intended to foster the dissemination of state-of-the-art research in the area of UIC and present exciting enabling opportunities/technologies. To this end, this special issue includes eight extended versions of selected papers originally presented at the 7 th international

C.-H. Hsu ( $\square)$

Department of Computer Science and Information Engineering, Chung Hua University, Hsinchu, Taiwan

e-mail: chh@chu.edu.tw conference on Ubiquitous Intelligence and Computing (UIC 2011), held in Xian, China. The papers selected for this issue not only contribute valuable insights and results, but also have particular relevance to ubiquitous multimedia, services, environments, systems, objects and safety aspects. They all present high quality results for tackling problems arising from the ever-growing fields of ubiquitous intelligence computing.

Ivan Lee and Ling Guan in their paper entitled "InterFrame Dependency in Multi-view Multi-Description Video Streaming" investigates video frame dependencies in multi-view multiple description video streaming. Interframe video dependencies for single-path and multi-path video streaming are analyzed. Numerical evaluation demonstrates that inter-dependency between viewpoint helps improving the coding efficiency at the cost of coding delay and streaming performance. Comparing the impact of different state transition probability of the evaluation model, it is found that ensuring successful transmission of subsequent video frames is more important than ensuring a rapid recovery from error states.

The paper by Simen Hagen and Frode Eika Sandnes entitled "Visual Scoping and Personal Space on Shared Tabletop Surfaces" explores a shared surface prototype for collaborative work that allows information hiding and personal space for individual participants. The approach allows the participants in a meeting or conference the ability to remove information of no interest, or add information as it becomes relevant. Using this technique, two or more participants can work together on the same surface, without being disturbed by the actions of the other participants. As the technique allows certain display areas to be used for personal space simultaneously by the participants, the overall available display real estate is exploited more efficiently. 
The paper by K.L. Eddie Law and Sunny So entitled: "QoS Control Framework for Content Satisfaction in Ubiquitous Multimedia Computing", an agent-based QoS control framework for delivering satisfactory multimedia traffic across the Internet is designed. The framework is currently built on top of existing networking protocols. The major components in the platform consists of ingress and egress agents. QoS monitoring capsules are regularly sent between agents in order to enable the ingress agent to adapt the content information, while meeting the requirements and expectations of subscribers and end users. Through multimedia content classification, whether it is for realtime or non-real-time, important or unimportant, traffic can be sent to subscribers to meet their expected multimedia quality in our framework.

The paper by Chao Chen entitled "System-wide Support for Safety in Pervasive Spaces" addresses issues of safety in pervasive spaces. The author raises awareness of the issue of safety in pervasive systems and its ties to the programming model used to program the pervasive applications. The author demonstrated the safety problem through a few motivating scenarios and discussed the new elements that needs to be addressed by any new programming models to address safety. They presented a redefinition of pervasive systems in terms of four elements and their safety requirements. The author also presented a redefinition and extensions of the necessary roles that participate in the creation and development of a pervasive space. The author proposed a model that utilizes rolespecific safety knowledge, and that takes advantage of the rich sensing and actuations capabilities of pervasive systems to detect and handle "conflicting contexts" and prevent or detect/avert "impermissible contexts". How does it mitigate overall safety risks in presence of uncertainty due to multiple independent roles is also presented.

The paper by Akifumi Sokan, Ming Wei Hou, Norihide Shinagawa, Hironori Egi and Kaori Fujinami entitled "A Tangible Experiment Support System with Presentation Ambiguity for Safe and Independent Chemistry Experiments" proposed and prototyped a tangible experiment support system named A3 for safe and independent chemistry experiments. The system presents information with three-dimensional ambiguity. The results of a usability test indicate that the participants generally perceived the system as a positive supplement. The analysis by another questionnaire and interviews showed that a major concern in the usability was improper context recognition.

The paper by Yue-Shan Chang, Chih-Tien Fan and Tong-Ying Juang entitled "Supporting software intelligence in ubiquitous environment exploits mobile agent" presented an integrated and flexible framework that can satisfy various applications in which the functionality of mobile agent is distinguishable in diversity of networks or systems. An agent-based platform that can guide various mobile agents that are initiated by mobile devices to complete their goal is introduced. In order to adapt various applications and simplify the implementation of mobile agents, in their implementation, context sensing module was separated from ordinary mobile agents. Several significant advantages can be obtained from the proposed architecture.

The paper by Jiehan Zhou, Junzhao Sun, Kumaripaba Athukorala, Dinesh Wijekoon and Mika Ylianttila entitled "Pervasive Social Computing: Augmenting Five Facets of Human Intelligence" reviews Pervasive Social Computing as an integrated computing environment, which promises to augment five facets of human intelligence: physical environment awareness, behaviour awareness, community awareness, interaction awareness, and content awareness. Reviews of related studies are given, and their generic architectures are designed. To have an explicit understanding of Pervasive Social Computing, the authors analyze its evolution and its links with other emerging computing technologies. They also present the generic architectures of these systems, then explore how these computing services augment awareness of community, physical environment, relevant content, and interactionoriented human intelligence.

The paper by Hongbo Ni, Bessam Abdulrazak, Daqing Zhang, Shu Wu, Zhiwen Yu, Xingshe Zhou and Shengrui Wang entitled "Towards Non-intrusive Sleep Pattern Recognition in Elder Assistive Environment" proposed an unobtrusive sleep pattern recognition system based on a kind of ultra-thin pressure sensor matrix. They presented the design of sensors deployment and the implementation of the sleep behaviour detection system. Moreover, based on the experiments, the authors discussed the data analysis and evaluation of the system and the result proved that the proposed solution is a promising way to monitor the elder's sleep postures and recognize his sleep pattern.

All of the above papers address either theoretical models, implementation and security issues in pervasive computing systems or propose novel application models in the various UIC fields. They also trigger further related research and technology improvements in application and services of UIC. Honorably, this special issue serves as a landmark source for education, information, and reference to professors, researchers and graduate students interested in updating their knowledge about or active in ubiquitous intelligence computing and novel application models for pervasive computing systems.

The guest editor would like to express sincere gratitude to Dr. Vincenzo Loia (AIHC, EIC), for giving me the opportunity to prepare this special issue. In addition, I am deeply indebted to numerous reviewers for their 
professional effort, insight and hard work put into commenting on the selected articles which reflect the essence of this special issue. Last but not least, I am grateful to all authors for their contributions and for undertaking twocycle revision of their manuscripts, without which this special section could not have been produced. 